\title{
Fertility sparing surgery in young women affected by endometrial stromal sarcoma: an oncologic dilemma or a reliable option? review of literature starting from a peculiar case
}

\author{
This article was published in the following Dove Press journal: \\ OncoTargets and Therapy \\ 18 December 2014 \\ Number of times this article has been viewed
}

\section{Marco Noventa \\ Salvatore Gizzo \\ Lorena Conte \\ Angela Dalla Toffola \\ Pietro Litta \\ Carlo Saccardi}

Department of Woman and Child Health, University of Padua,

Padua, Italy
Correspondence: Salvatore Gizzo

Dipartimento della Salute della Donna

e del Bambino, UOC di Clinica

Ginecologica e Ostetrica Via Giustiniani

3, 35I 28 Padova, Italy

Tel +39 $3335727248,+390498213400$

Fax $+39049821 \quad 1785$

Email ginecologia_padova@libero.it
Background: Endometrial stromal sarcoma (ESS) is a term used to define a rare neoplasm that accounts for approximately $0.2 \%-1 \%$ of all uterine malignancies; it is, however, implicated in an estimated $10 \%-15 \%$ of those malignancies with a mesenchymal component. Recent evidence suggests that while the preservation of the ovaries may be considered appropriate in premenopausal women, hysterectomy and bilateral salpingo-oophorectomy remains the recommended treatment in postmenopausal women. Currently, only a few case series reporting the treatment of ESS in young women with a desire to preserve fertility and thus subjected to a fertility-sparing surgery are available in the literature.

Case presentation: We report a peculiar case of early stage ESS treated by laparoscopic fertility-sparing surgery and a strict follow-up program (every 3 months) of imaging and clinical evaluation. The patient remained disease free 1 year after primary treatment. Three months after completing oncological follow-up, the patient conceived spontaneously and is, to date, pregnant at 11 weeks of gestation without evidence of recurrent disease or obstetric complications.

Conclusion: Based on our case report and in accordance with the data available, we suggest that in young patients affected by early stage ESS who wish to preserve reproductive function, fertility-sparing surgery could represent a valid option, though strict oncological follow-up remains mandatory.

Keywords: young women, laparoscopic surgery, pregnancy, neoplasia, surgical management, follow up, disease recurrence

\section{Background}

Uterine sarcomas are rare neoplasms that originate from either the connective tissue elements in the endometrium or from the myometrium. ${ }^{1-3}$ The term endometrial stromal sarcoma (ESS) is applied to rare neoplasms that comprise approximately $0.2 \%-1 \%$ of all uterine malignancies and $10 \%-15 \%$ of uterine malignancies with a mesenchymal component. $^{1,4,5}$

This neoplasm is typically composed of cells resembling the endometrial stromal cells of a proliferative endometrium. ${ }^{1,4}$ According to biological behaviors, they may be classified into noninvasive (noninvasive/stromal nodules) and invasive (low-grade ESS). ${ }^{1,4}$

The typical clinical manifestation of ESS is as a uterine intracavitary polyp(s) or intramural mass(es), often characterized by ill-defined borders and signs of myometrial infiltration. It shows only mild nuclear atypia and rarely tumor cell necrosis; a breakpoint of ten mitoses per ten high-power fields was used to distinguish between low-grade and high-grade ESS. ${ }^{1}$ 
Although ESS pathogenesis is poorly understood, specific cytogenetic aberrations and molecular changes have been recently elucidated. ${ }^{4-6}$ In particular, almost all ESS are characterized by an overexpression of estrogen and progesterone receptors, reported in approximately $70 \%$ and $95 \%$ of cases, respectively. ${ }^{5-7}$

While recent evidence suggests that in premenopausal women younger than 35 years age presenting with a small primary tumor $(<2-3 \mathrm{~cm})$ the preservation of ovaries may be appropriate, in postmenopausal women the recommended treatment remains total hysterectomy and bilateral salpingooophorectomy (BSO).,7

In young patients with early stage ESS, the preservation of ovarian function without an associated increase in oncological risk allows us to consider the prospect of preservation of reproductive function by implementation of fertilitysparing surgical techniques, currently successfully applied as treatment in other gynecological malignancies, to highly selected patients in the abovementioned group. ${ }^{8,9}$ Only a few case series are available in the literature concerning ESS treatment in young women who desire a future pregnancy subjected to fertility-sparing surgery. ${ }^{10-15}$

Based on these considerations, it seems mandatory to define, without delay, the best surgical approach, the timing of the surgical procedure, and the role of adjuvant therapy in order to achieve the best oncological outcome without affecting subsequent fertility rate in this selected cohort of patients.

The aim of this report is to review current available literature concerning the appropriate management of early stage ESS diagnosed in young patients who desire future pregnancy as beginning from our very peculiar case: a stage I ESS treated by laparoscopic conservative surgery. The oncological follow-up consists of both imaging techniques and a relaparoscopic/hysteroscopic evaluation performed 1 year after the initial surgical treatment.

\section{Case presentation}

A 34-year-old nulliparous woman was referred to our unit (Minimally Invasive-Pelvic-Surgery Unit-Woman and Children's Health Department, University of Padua) with the following clinical presentation: hypermenorrhea, abdominal/ pelvic pain, and progressively worsening anemia (past 6 months). The patient's medical and surgical histories were unremarkable, as were previous Pap smears and pelvic examinations. Abdominal and gynecological examination revealed an enlarged uterus of irregular shape due to the presence of a nontender abdominal mass that could be referred to as a leiomyoma. No adnexal masses were detected. Transvaginal sonography (TVS) confirmed our diagnostic hypothesis and described a hypoechoic uterine mass $(7.7 \times 6.2 \times 5.8 \mathrm{~cm}$ located in the anterior-left uterine wall extending to the endometrial surface without abnormal vascularization pattern) suggestive for leiomyoma.

Preoperative magnetic resonance imaging (MRI) confirmed the presence of a voluminous uterine mass, with heterogeneous signal intensity and early intense enhancement after contrast injection, involving the endometrial cavity and an absence of a well-defined endometrial-myometrial junction. No evidence of retroperitoneal lymphadenopathy, intraperitoneal disease, or regional metastases was shown (Figure 1A and B). Hysteroscopic investigation was negative for intrauterine benign or malignant lesions. We planned surgical laparoscopic myomectomy. At laparoscopic exploration, the pelvis appeared unremarkable, without signs of extrauterine disease. Adnexa appeared normal; the uterus presented irregularities in morphology, but no signs of serosal involvement were found (Figure $2 \mathrm{~A}-\mathrm{C}$ ).

We proceeded with enucleation of the uterine mass according to our previously described technique. ${ }^{16}$ Due to the intermediate soft-hard consistency of the leiomyoma, a frozen section histology was performed which showed benign-appearing smooth muscle proliferation with no cellular atypia or excessive mitotic activity. Given the age of the patient, the lack of pelvic and lymph-vascular disease involvement, and the negative frozen section histology, a conservative surgical approach was chosen. The complete enucleation of the intramural mass required the opening of the uterine cavity. Due to the persisting suspicion of an unusual uterine lesion, we performed an accurate minimal morcellation of the lesion and extraction of the specimen by endobag. The endometrial cavity was then repaired and the myometrium was reconstructed in three layers using Size 0 Polysorb $^{\text {TM }}$ Suture (Polysorb, USSC, Norwalk, CT, USA). Multiple abdomen/pelvis washing with warm sterile water was performed at the end of the surgical procedure. The subsequent hospital stay showed no signs of surgical complications, and 2 days later the patient was discharged.

The definitive histology reported a diagnosis of ESS with minimal nuclear pleomorphism, low mitotic index, and an infiltrative growth pattern within the myometrium. Immunohistochemical investigation found cells positive for the presence of CD10, muscle-specific actin, and vimentin, whereas it tested negative for desmin and h-caldesmon. There was strong nuclei antibody staining for estrogen and progesterone receptors (Figure $3 \mathrm{~A}-\mathrm{D}$ ). 

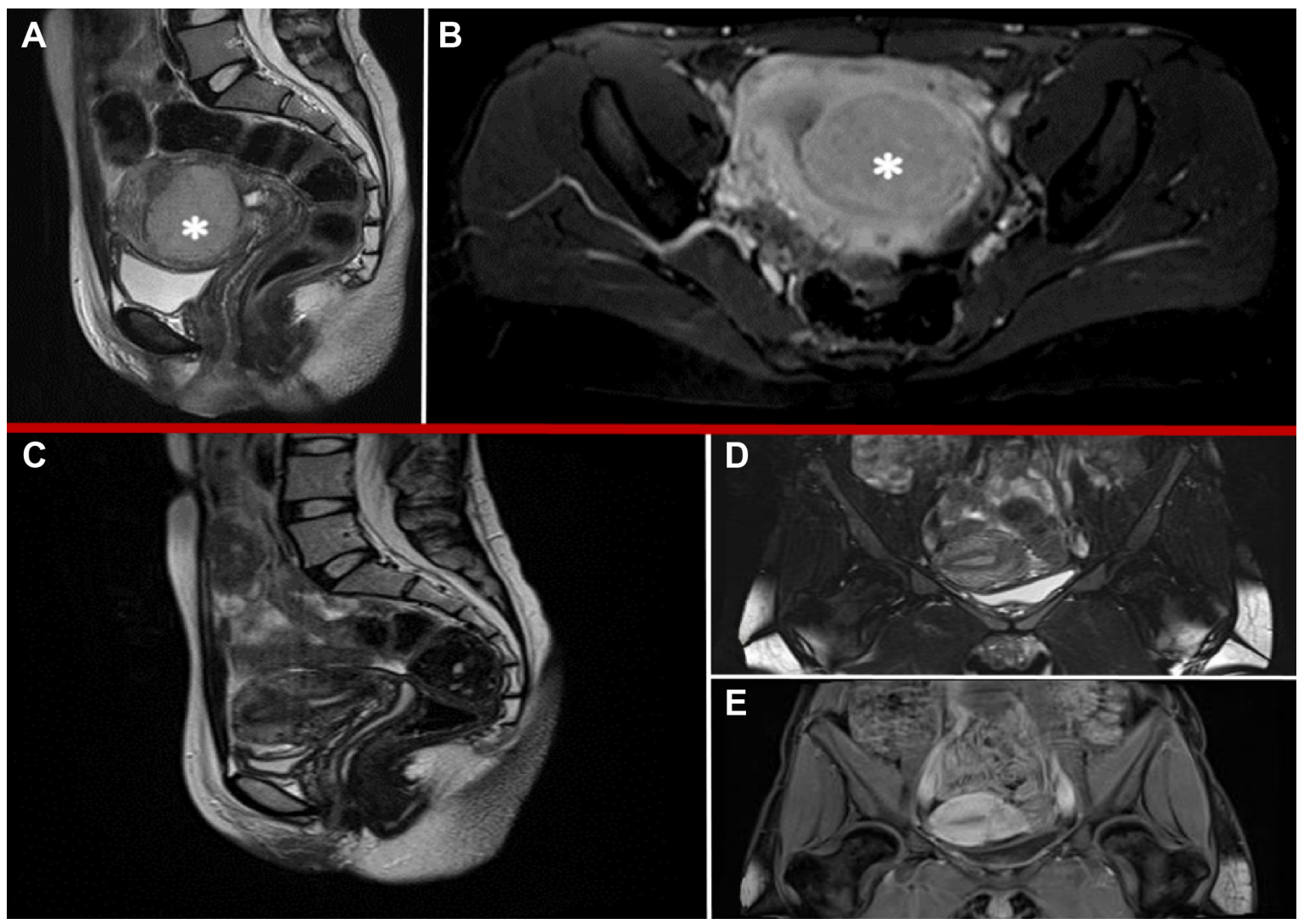

Figure I MRI features: preoperative (A, B) and 6 months after surgery (C-E).

Notes: (A) Saggital-T2 weighted imaging of well-defined uterine lesion with dishomogeneous signal intensity. (B) Axial-T2 weighted imaging with contrast enhancement showing early intense strengthening in the postenhancement phase. (C) Saggital-T2 weighted imaging of regular uterus. (D) Coronal-T2 weighted imaging of regular uterus.

(E) Coronal-TI weighted imaging with low-contrast enhancement in area of previous surgery. *indicates the uterine lesion.

Abbreviation: MRI, magnetic resonance imaging.
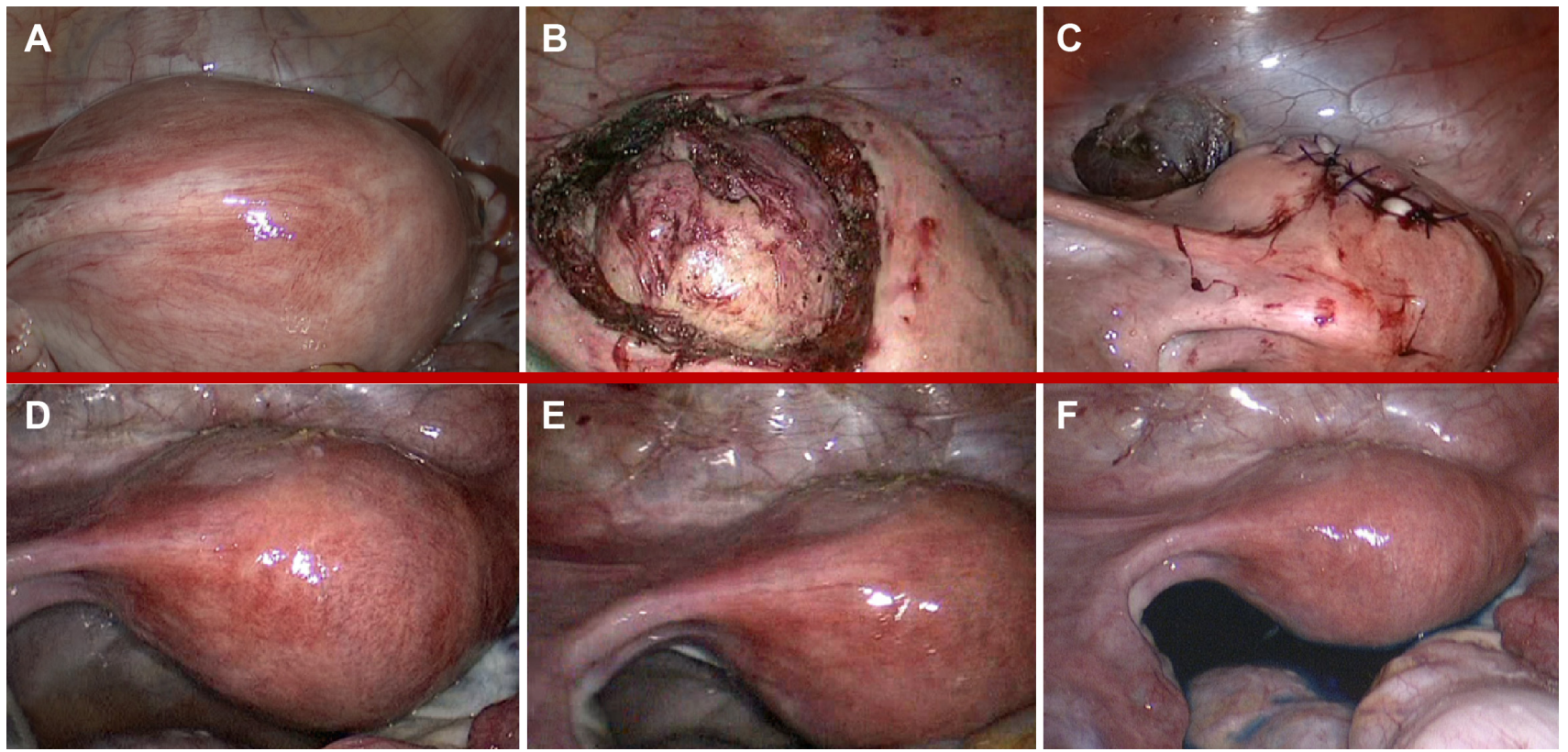

Figure 2 Laparoscopic features: intraoperative (A-C) and 6 months after surgery (D-F).

Notes: (A) Panoramic view of intact uterus before the ESS enucleation. (B) Panoramic view of ESS during enucleation. (C) Panoramic view of uterus after ESS enucleation. (D-E) Panoramic view of disease-free pelvis and uterus. (F) Cromosalpingoscopy with direct visualization of bilateral transtubal methylene spillage.

Abbreviation: ESS, endometrial stromal sarcoma. 

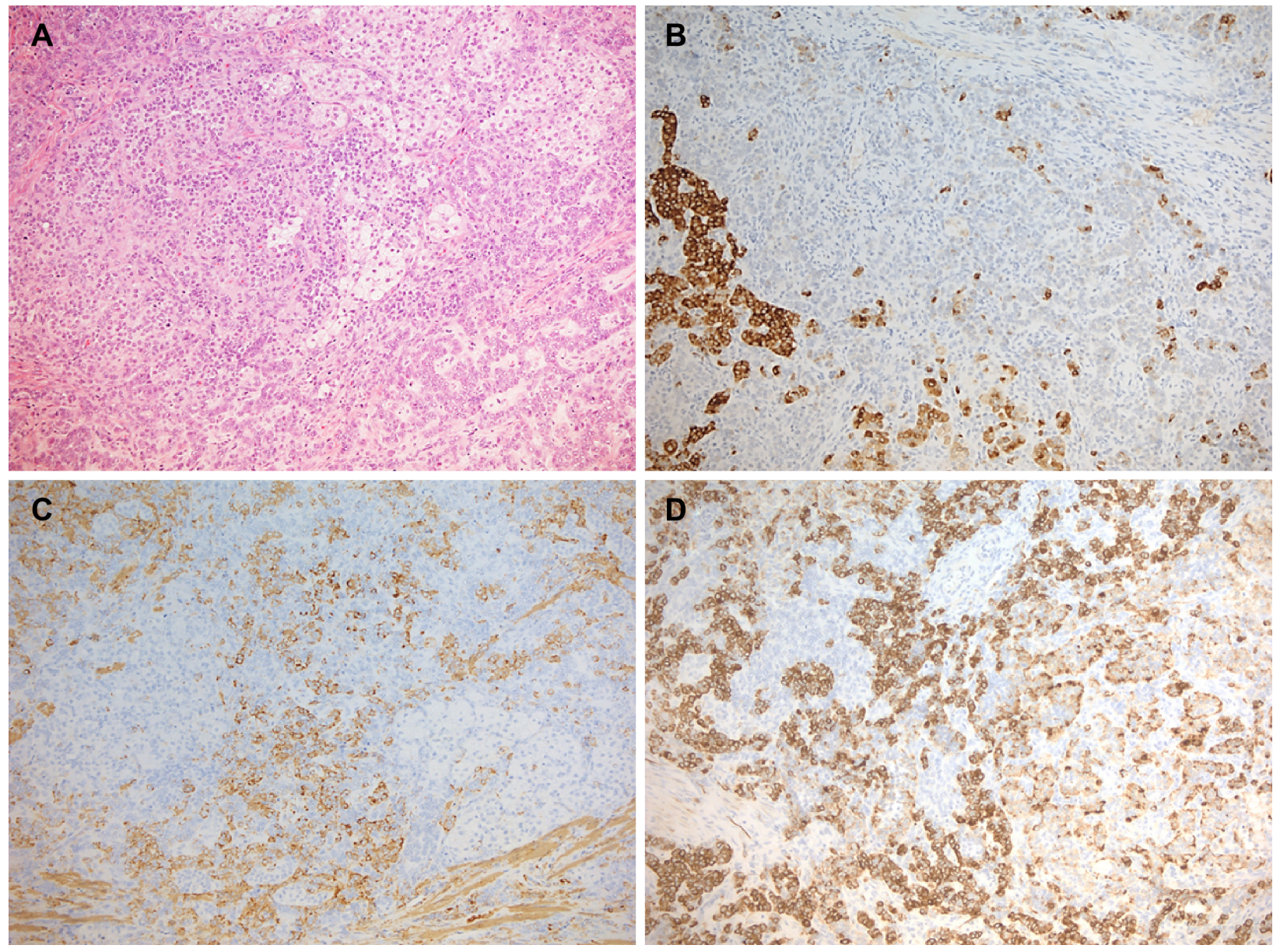

Figure 3 Microscopic appearance with the appropriate pathological specimen description.

Notes: (A) The tumor showed an admixture of neoplastic cells arranged in cords and trabeculae with an island of lipidized cells. (B) These vacuolated cells were positive for inhibin, whereas the others (C) were strongly immunoreactive for SMA. In both components, MNFI I6 was widely positive (D). (Original magnification I0X). Abbreviation: SMA, smooth muscle actin.

Counseling with the patient regarding histological findings and the risk of recurrence was performed. We offered second-look surgery and oncological counseling in order to discuss the possibility of adjuvant therapy. Due to the patient's strong desire for preserving fertility and the negativity of all oncological serum markers (CA125, CEA, CA19.9, CA15.3, HE4), we decided on a conservative approach, postponing surgical and adjuvant therapy until after the planned pregnancy.

Sequential clinical examination and diagnostic imaging was performed every 3 months in postoperative surveillance. TVS and MRI showed normal uterine size and vascularization pattern; no evidence of intraperitoneal disease or pelvic and para-aortic lymph node involvement was detected 6 months after surgery (Figure 1C-E).

In order to exclude a possible misdiagnosed recurrence (linked to shorter progression free-survival described in literature associated with morcellation of the mass) ${ }^{7}$ we performed, 7 months after the first surgery, hysteroscopic evaluation and multiple endometrial biopsies (all negative for disease recurrence) and a diagnostic laparoscopic second-look which did not show any macroscopic evidence of malignancy, subsequently confirmed by cytological examination of pelvic peritoneal washing (Figure 2D-F).

One year after conservative surgical treatment, the patient showed good clinical condition, return of regular menses, and no evidence of recurrences. Three months after the last oncological follow-up, the patient conceived spontaneously and, to date, is pregnant at 11 weeks of gestation without evidence of recurrent disease or obstetric complications.

\section{Conclusion}

ESS is a rare hormone-sensitive uterine malignancy that typically affects women both of reproductive and postmenopausal age (major incidence between 40-55 years; annual incidence one to two per million women). The typical clinical 
manifestation of the disease is vaginal bleeding, in some cases associated with pelvic/abdominal pain. ${ }^{1-6}$ Although clinical symptoms present early, the majority of ESSs are misdiagnosed. Often, routine imaging (TVS, MRI) seems unable to discriminate between leiomyoma and ESS; for this reason, preoperative diagnosis is not easy. ${ }^{6,17}$ This neoplasm can be diagnosed preoperatively through hysteroscopic biopsies or through endometrial curettage. ${ }^{7,18}$ Intraoperative frozen section seems to have an intermediate diagnostic accuracy; only definitive histopathology examination achieves a good accuracy, thanks to the invaluable contribution of immunohistochemistry investigations..$^{4,7}$

The standard treatment proposed for ESS is laparotomic total hysterectomy and BSO with or without pelvic lymphadenectomy, ${ }^{7}$ even if, in highly selected patients, a robotic or laparoscopic approach is discussed. ${ }^{7,19,20}$ Although the hysteroscopic approach showed good consistency in terms of surgical efficacy and fertility outcome in the event of intrauterine benign lesions $\mathrm{s}^{21,22}$ it cannot be recommended for small intrauterine ESS due to the inability of obtaining information regarding the adnexa and peritoneum.

The main doubts concerning the standard surgical procedure proposed are related to the indication of both lymphadenectomy and BSO: first of all, there is no evidence of increased survival in patients who underwent complete lymphadenectomy compared to women who did not have this procedure as part of their surgery. ${ }^{7}$ Regarding BSO, we can suppose that an ovary-sparing surgery could not be recommended for an estrogen-sensitive neoplasm; however, recent evidences by Chan et al demonstrated that in highly selected patients younger than 50 years with early stage (International Federation of Gynecology and Obstetrics [FIGO] stage I-II) ESS, ovarian sparing surgery did not affect overall survival. ${ }^{23}$

Generally, ESS showed a good 10-year survival rate for both early and advanced stages of disease (from $89 \%$ in stage I to $66 \%$ in stage IV). However, recurrences can occur from 3 months to 23 years after treatment, with a median interval of approximately 3 years. ${ }^{7,14}$ The most important prognostic factors reported on multivariate analysis are FIGO stage, tumor diameter, menopausal status, tumor grade, and tumor-free resection margins. In particular, this last parameter represents the single most important oncological prognostic factor. ${ }^{4,5,7,17}$

Considering the good prognosis of ESS, several authors proposed conservative management to preserve fertility in young women, and rare cases of pregnancy after fertility sparing surgery have been reported. ${ }^{10-15}$ Stadsvold et al described a case of a nulliparous 16-year-old adolescent girl managed by laparotomic complete resection of uterine mass (negative for neoplasia at intraoperative frozen section) subsequently described as early stage ESS. Due to the patient's young age and her desire to preserve future fertility, the uterus was preserved and the patient treated with a cycle of megestrol acetate as adjuvant therapy. The patient showed no evidence of recurrent disease 21 months after surgery. ${ }^{12}$ Koskas et al in 2009 reported a case of a 34-year-old woman conservatively treated through a hysteroscopic resection of a polypoid endometrial mass which subsequently proved to be an ESS (with no suspicion of pelvic dissemination). She conceived a healthy boy 6 months after surgical treatment. In the postpartum period, the persistence of pelvic pain prompted the performance of a MRI and a subsequent laparoscopic exploration, which revealed the presence of a bilateral pelvic mass with peritoneal recurrence. ${ }^{14}$

Sanchez-Ferrer et al described a case of disease recurrence after conservative treatment for ESS. Initially, the patient underwent a laparotomic surgical procedure for a leiomyoma. Though the definitive histological examination showed the typical pattern of ESS with infiltrated margins, the patient declined any type of repeat surgery before attempting pregnancy. The patient conceived after in vitro fertilization and delivered a healthy baby weighing $32 \mathrm{~g}$. During the postpartum period, due to persistent suspicion of disease recurrence at imaging, the woman consented to undergo second-look surgery. Total hysterectomy without adnexectomy was performed and a definitive diagnosis of stage-IB ESS was attained. ${ }^{11}$

Contrasting this last report, Yan et al described an uneventful physiologic pregnancy in a 25 -year-old woman who underwent conservative management for ESS. After the first postoperative diagnosis of early stage ESS and prior to pregnancy, the patient underwent a second-look surgical procedure with the excision of the area of fusiform myometrium extending $3 \mathrm{~cm}$ adjacent to the former uterine incision to the submucous layer. Interestingly, 5 years after the first diagnosis, the authors described no signs of any further recurrences. ${ }^{13}$

Delaney et al described an optimal outcome for a premenopausal patient subjected to a conservative management after postoperative diagnosis of ESS. ${ }^{10}$ The patient received adjuvant therapy through high-dose megestrol acetate. The authors reported no evidence of recurrences 8 years after the diagnosis; subsequently, the patient delivered a live born neonate through Cesarean section. ${ }^{10}$ 
In our report, we described a laparoscopic surgical approach for an unsuspected ESS treated as a benign leiomyoma. All preoperative imaging techniques and intraoperative microscopic analysis showed no evidence of malignancies. Considering that inadvertent tumor morcellation is associated with a shorter progression-free survival in patients with ESS, we advised the patient to perform second-look surgery. ${ }^{4,7}$ Our patient denied the proposed surgical treatment and preferred to undergo strict follow-up as previously reported. As of today, there is no evidence of recurrence or disease relapse.

In the current literature, there are poor evidences concerning the fertility-sparing surgical approach for ESS. Therefore, it remains difficult to ascertain clear evidence on safety and effectiveness of conservative management. It is also evident that we are speculating with regards to a scarcely aggressive neoplasm which often occurs in young premenopausal women in whom the desire of offspring is not yet satisfied. Based on these premises, it is mandatory to define more strict criteria for the surgical management of such an illness, particularly in young patients who strongly desire pregnancy. Following a definitive diagnosis of ESS, adequate counseling regarding the risks and benefits of the therapeutical alternatives remains the most important step in defining future surgical and medical strategies.

There remain unanswered questions which may aid surgeons in defining the cohort of young women with a low oncological recurrence risk who are more likely to benefit from fertility-sparing surgery. As mentioned above, FIGO stage, tumor diameter, tumor grade, and tumor-free resection margins are the most important prognostic factors for ESS. Recently, the expression of the immunohistochemical marker CD10 on ESS cell surface has been suggested as a positive prognostic marker. ${ }^{17}$ Different authors suggest that ovarysparing surgery may represent a safe option for patients characterized by early stage disease (FIGO I-II), small primary tumor, and free resection margins. ${ }^{4,7}$ According to the evidence stated, we can suppose that these strict criteria could be a useful starting point to define the feasibility and efficacy of a conservative management for ESS. We emphasize the fact that evidence of absence of extrauterine or peritoneal disease determined by ultrasonography, MRI, and finally abdominal exploration are fundamental points in order to propose a fertility-sparing surgery.

The role of adjuvant therapy after a conservative surgical approach for ESS is still not defined. Chemotherapy and radiation therapy has a proven efficacy as second-line treatment for patients with recurrent or metastatic disease. ${ }^{4,7}$
The first-line treatment with proven efficacy, considering that ESSs overexpress both estrogen and progesterone receptors, is progesterone-like and antiestrogenic agents such as megestrol, GnRH analogues, and aromatase inhibitors. ${ }^{4,7}$ However, the available data is unable to define specific protocols on treatment duration and hormone regimens after a fertility-sparing procedure. More importantly, it is necessary to understand and define the exact timing of adjuvant therapy and pregnancy development. In the recent paper by Lissoni et al the authors postulate that "the increased level of steroid hormones during pregnancy might even result in a protective role against further development of the tumor." 15

It remains unclear whether it is advantageous (to decrease the oncological recurrence risk) to perform, immediately following surgery, a hormone treatment cycle with a subsequent delay of pregnancy as opposed to instituting an adequate surveillance protocol while awaiting completion of the reproductive cycle prior to evaluating the possibility of adjuvant therapy or second-look surgery. Recent evidence, in agreement with our case report, suggests that in highly motivated and strictly selected patients, fertility-sparing surgery is possible and likely associated with a very low risk of disease relapse. Adequate and strict follow-up in a gynecological oncology center with a dedicated team is mandatory. To further lower the recurrence rate, radical surgery may be proposed following the completion of the woman's reproductive cycle.

\section{Acknowledgments}

We wish to thank the Department of Pathology for aiding us in collecting data regarding the pathological specimen and in formulating a diagnosis, the nursing staff for caring for the patient during the follow-up period, and Dr Di Gangi Stefania for her invaluable contribution in translation of the manuscript into English.

This type of manuscript (case report with literature review) does not require approval by the local Ethical Committee (Padua Ethical Committee). However, as routinely occurs, patients who undergo treatment in a public Health Care Unit consent, upon hospital admission, to the use of their personal data in respect of Italian privacy law (196/94) for scientific use and divulgation. We obtained written consent to anonymously divulgate data regarding the management of the clinical case.

\section{Author contributions}

All authors contributed toward data analysis, drafting and revising the paper and agree to be accountable for all aspects of the work. 


\section{Disclosure}

The authors report no conflicts of interest in this work.

\section{References}

1. Tavassoli FA, Devilee P. World Health Organization Classification of Tumors. Pathology and genetics: tumors of the breast and female genital organs. IARC Press: Lyon, France; 2003.

2. Patrelli TS, Gizzo S, Di Gangi S, Guidi G, Rondinelli M, Nardelli GB. Cervical Mullerian adenosarcoma with heterologous sarcomatous overgrowth: a fourth case and review of literature. BMC Cancer. 2011;11:236

3. Patrelli TS, Silini EM, Gizzo S, et al. Extragenital Müllerian adenosarcoma with pouch of Douglas location. BMC Cancer. 2011;11:171.

4. Tropé CG, Abeler VM, Kristensen GB. Diagnosis and treatment of sarcoma of the uterus. A review. Acta Oncol. 2012;51(6):694-705.

5. Prat J. FIGO staging for uterine sarcomas. Int J Gynaecol Obstet. 2009; 104(3):177-178.

6. Tirumani SH, Ojili V, Shanbhogue AK, Fasih N, Ryan JG, Reinhold C. Current concepts in the imaging of uterine sarcoma. Abdom Imaging. 2013;38(2):397-411

7. Rauh-Hain JA, del Carmen MG. Endometrial stromal sarcoma: a systematic review. Obstet Gynecol. 2013;122(3):676-683.

8. Gizzo S, Ancona E, Saccardi C, et al. Radical trachelectomy: the first step of fertility preservation in young women with cervical cancer (Review). Oncol Rep. 2013;30(6):2545-2554.

9. Gizzo S, Ancona E, Patrelli TS, et al. Fertility preservation in young women with cervical cancer: an oncologic dilemma or a new conception of fertility sparing surgery? Cancer Invest. 2013;31(3):189.

10. Delaney AA, Gubbels AL, Remmenga S, Tomich P, Molpus K. Successful pregnancy after fertility-sparing local resection and uterine reconstruction for low-grade endometrial stromal sarcoma. Obstet Gynecol. 2012;120(2 Pt 2):486-489.

11. Sánchez-Ferrer ML, Machado-Linde F, Ferri-Ñíguez B, Sánchez-Ferrer M, Parrilla-Paricio JJ. Reproductive outcome after uterine-sparing surgery for endometrial stromal sarcoma. Gynecol Oncol Case Rep. 2012;3:4-6.

12. Stadsvold JL, Molpus KL, Baker JJ, Michael K, Remmenga SW. Conservative management of a myxoid endometrial stromal sarcoma in a 16-year-old nulliparous woman. Gynecol Oncol. 2005;99(1):243-245.
13. Yan L, Tian Y, Fu Y, Zhao X. Successful pregnancy after fertilitypreserving surgery for endometrial stromal sarcoma. Fertil Steril. 2010; 93(1):269.e1-e269.e3.

14. Koskas M, Morice P, Yazbeck C, Duvillard P, Walker F, Madelenat P. Conservative management of low-grade endometrial stromal sarcoma followed by pregnancy and severe recurrence. Anticancer Res. 2009; 29(10):4147-4150.

15. Lissoni A, Cormio G, Perego P, Gabriele A, Cantú MG, Bratina G. Conservative management of endometrial stromal sarcoma in young women. Int J Gynecol Cancer. 1997;7(5):364-367.

16. Pluchino N, Litta P, Freschi L, et al. Comparison of the initial surgical experience with robotic and laparoscopic myomectomy. Int $J$ Med Robot. 2014;10(2):208-212.

17. He L, Li JD, Xiong Y, et al. Clinicopathological and molecular markers associated with prognosis and treatment effectiveness of endometrial stromal sarcoma: a retrospective study in China. Arch Gynecol Obstet. 2014;289(2):383-391.

18. Litta P, Merlin F, Saccardi C, et al. Role of hysteroscopy with endometrial biopsy to rule out endometrial cancer in postmenopausal women with abnormal uterine bleeding. Maturitas. 2005;50(2):117-123.

19. Litta P, Fantinato S, Calonaci F, et al. A randomized controlled study comparing harmonic versus electrosurgery in laparoscopic myomectomy. Fertil Steril. 2010;94(5):1882-1886.

20. Litta P, Saccardi C, Conte L, Florio P. Reverse hysterectomy: another technique for performing a laparoscopic hysterectomy. J Minim Invasive Gynecol. 2013;20(5):631-636.

21. Saccardi C, Conte L, Fabris A, et al. Hysteroscopic enucleation in toto of submucous type 2 myomas: long-term follow-up in women affected by menorrhagia. J Minim Invasive Gynecol. 2014;21(3):426-430.

22. Litta P, Conte L, De Marchi F, Saccardi C, Angioni S. Pregnancy outcome after hysteroscopic myomectomy. Gynecol Endocrinol. 2014; 30(2):149-152.

23. Chan JK, Kawar NM, Shin JY, et al. Endometrial stromal sarcoma: a population-based analysis. Br J Cancer. 2008;99(8):1210-1215.
OncoTargets and Therapy

\section{Publish your work in this journal}

OncoTargets and Therapy is an international, peer-reviewed, open access journal focusing on the pathological basis of all cancers, potential targets for therapy and treatment protocols employed to improve the management of cancer patients. The journal also focuses on the impact of management programs and new therapeutic agents and protocols on

\section{Dovepress}

patient perspectives such as quality of life, adherence and satisfaction. The manuscript management system is completely online and includes a very quick and fair peer-review system, which is all easy to use. Visit http://www.dovepress.com/testimonials.php to read real quotes from published authors. 